\title{
Modulatory effect of luteolin on redox homeostasis and inflammatory cytokines in a mouse model of liver cancer
}

\author{
QIANG ZHANG, JIE YANG and JUN WANG
}

\author{
Department of General Surgery, Xiangyang Hospital, Affiliated Hospital of Hubei University of Medicine, \\ Xiangyang, Hubei 441000, P.R. China
}

Received December 31, 2014; Accepted February 24, 2016

DOI: 10.3892/ol.2016.5291

\begin{abstract}
Liver cancer is one of the leading causes of cancer-associated mortality worldwide. Due to changes in lifestyle and daily exposure to various chemicals, which may lead to chemical intoxication, liver cancer has become a prominent disease in humans. Chemical-induced carcinogenesis in experimental animals has become a reliable model for the investigation of liver cancer-associated biological alterations that may mimic human hepatic cancer. Liver cancer in BALB/c mice was induced by administering diethylnitrosamine (DN) in drinking water for 6 weeks. Luteolin (LUT) is a flavone that is found in the leaves of the majority of spice-associated plants. In the present study, $20 \mu \mathrm{g} / \mathrm{kg}$ of body weight LUT was administered intraperitoneally every alternate day to treat the DN-induced liver cancer in mice. LUT improved the host system by modifying the levels of $\alpha$-fetoprotein, enzymatic antioxidants, such as superoxide dismutase and catalase, marker enzymes, such as AST and ALT, and lipid peroxides in the plasma or liver tissue. LUT also reduced the levels of glutathione and the inflammatory cytokines interleukin-2 and interferon- $\gamma$ in the plasma or liver tissue. These findings augmented the treatment against liver cancer and supported the effective anticancer activity of LUT against DN-induced liver carcinogenesis in mice.
\end{abstract}

\section{Introduction}

Liver cancer accounts for $>5 \%$ of all cancers worldwide and is the third most common cause of mortality globally, being the fifth most common cause for men and eighth most common cause for women (1). Since the majority of patients with liver cancer are diagnosed at the end-stage of liver dysfunction, the

Correspondence to: Dr Qiang Zhang, Department of General Surgery, Xiangyang Hospital, Affiliated Hospital of Hubei University of Medicine, 15 Jiefang Road, Xiangyang, Hubei 441000, P.R. China

E-mail: qiangzhang128@gmail.com

Key words: liver cancer, diethylnitrosamine, luteolin, inflammatory cytokines, marker enzymes, lipid peroxidation mortality rate and incidence of liver cancer are similar (2). The course of malignant cancer transformation involves the accumulation of mutations and aberrations among the genes that govern proliferation and apoptosis in cells, leading to apoptotic avoidance, neo-angiogenesis and metastatic potential (3). During the multistep development process in mature hepatocytes, genetic alterations in the hepatocytic microenvironment lead to necrosis, inflammation and re-generation, resulting in the cell populations becoming dysplastic nodules that eventually develop into a prominent cancer (4). Currently, surgical resection is one treatment modality used to treat liver cancer that decreases the mortality rate to $<5 \%$, provided that liver function is maintained at satisfactory levels (5). Inhibitors of tyrosine kinases provide opportunities for curative resection when administered as a preoperative neoadjuvant therapy (6). Chemotherapeutic drugs, such as cyclophosphamide and busulphan, which are used as a conditioning regimen, have immunosuppressive and myeloablative effects and associated complications, including mucositis, liver toxicity and hemorrhagic cystitis $(7,8)$. In addition, the chemotherapy may increase lipid peroxidation (LPO) and malondialdehyde levels, leading to oxidative stress in patients (9). Superoxide dismutase (SOD), catalase (CAT) and reduced glutathione (GSH)-dependent enzymes contribute to the enzymatic antioxidant system of cells, which protects against reactive oxygen species (ROS) and oxidative damage (10). However, circulating tumor cells generally enter into the circulation and cause postoperative recurrence and metastasis through an extremely complex process (11).

Luteolin (LUT) is a naturally occurring polyphenolic flavone that is present in the form of glycosides in numerous fruits and vegetables. Epidemiological studies have suggested that a LUT-rich plant derived diet may play an important role in the reduction of numerous diseases through the pharmacological activity of LUT, including antioxidant, anti-inflammatory, antimicrobial, anticancer, anti-allergic and anti-platelet properties (12). LUT has been shown to have an inhibitory effect on the growth of human pancreatic cancer cells and was able to induce apoptosis of these cells, leading to DNA damage (13). Absorption at the skin surface and reaching deeper skin layers adds the ability of LUT to be used as an antiphlogistic agent (14). Absorption of ultraviolet (UV) light by LUT revealed the possibility of its use in sun protection products that may potentially prevent skin cancer and skin 
aging problems (15). Notably, food supplementation with LUT increased the sensitivity of cancer cells to chemotherapy (16). Since the beneficial effects of LUT have been documented in various diseases, including numerous cancers, the present study reports the anti-hepatocarcinogenic effects of LUT in diethylnitrosamine (DN)-intoxicated mice.

\section{Materials and methods}

Drugs and chemicals. DN, LUT, 5,5'-dithiobis-2-nitrobenzoic acid and malondialdehyde were purchased from Sigma-Aldrich (St. Louis, MO, USA). All other chemicals used for the assays were of the highest quality and analytical grade (Nanjing Jiancheng Bioengineering Research Institute, Nanjing, China).

Animal experiment. In total, 24 male BALB/c mice weighing $20 \pm 2 \mathrm{~g}$ were used for the experiments. The mice were procured from the Laboratory Animal Center of Wuhan University (Wuhan, Hubei, China) and were fed with standard pelleted feed and had free access to purified sterile drinking water. The mice were acclimatized and housed under conditions of controlled temperature with $12 \mathrm{~h}$ light and dark cycles. The present protocol for animal experimentation was approved by the Institutional Animal Care and Use Committee for Laboratory Experiments at Hubei University of Medicine (Xiangyang, Hubei, China).

The 6-week-old mice were divided into 4 groups, each containing 6 mice. Group I acted as the normal control group and group II acted as the drug control group, with an intraperitoneal administration of $20 \mu \mathrm{g} / \mathrm{kg}$ of body weight LUT on each alternate day between week 7 and the end of the experimental period at week 12. In a separate group of DN-intoxicated animals, various doses of LUT $(1,5,10,20$ and $50 \mu \mathrm{g} / \mathrm{kg}$ of body weight) were administered to optimize the dose for a maximum efficacy at a minimal dose (data not shown) and a dose of $20 \mu \mathrm{g} / \mathrm{kg}$ of body weight was finalized. Group III animals were administered with DN at a dose of $100 \mathrm{mg} / \mathrm{kg}$ of body weight every week, which was dissolved in sterile water and administered using oral gavage for a maximum of 6 weeks. Group IV animals were initially administered with DN, as in group III, for the first 6 weeks and were then administered with LUT treatment from week 7 onwards, as in group II, until the end of the experimental period. At the end of the experimental period, all the animals were sacrificed subsequent to overnight fasting for $\geq 12$ h. Plasma samples were collected for different assays and immediately stored at $-20^{\circ} \mathrm{C}$ until use. The collected liver tissues were immediately rinsed with ice-cold physiological saline $(0.9 \% \mathrm{NaCl}$; Sigma-Aldrich) and were processed for analysis.

Marker enzymes and enzymatic antioxidant assays. Assays for the measurement of the activity of AST and ALT in the plasma and tissue were performed using a UV-visible spectrophotometer (Lengguang Instrument Co., Ltd., Shanghai, China), according to the manufacturer's protocol. The activity of SOD and CAT was assayed using the manufacturer's instructions from Calbiochem, Inc. (San Diego, CA, USA) using a UV-visible spectrophotometer.
Assays for LPO and GSH. LPO was determined in the plasma and liver tissue using the thiobarbituric acid reaction, as previously described by Ohkawa et al (17). The level of lipid peroxides was expressed as mmol thiobarbituric acid reactive substances (TBARS)/ml of plasma or nmol TBARS/g of tissue. The GSH level in the plasma and liver was determined by the method previously reported by Ellman (18). The amount of GSH is expressed as $\mathrm{mg} / \mathrm{dl}$ of plasma or $\mathrm{mg} / \mathrm{g}$ of liver tissue.

Determination of the $\alpha$-fetoprotein $(A F P)$ level in the plasma. The AFP level was determined in the plasma using an Abbott chemiluminescence analyzer (Abbott Laboratories, Chicago, IL, USA) as per the manufacturer's instructions. The technique was based on immunoassay (19) and the amount of relative light units was measured and expressed as $\mathrm{ng} / \mathrm{ml}$.

Determination of the levels of IL-2 and IFN- $\gamma$ by enzyme-linked immunosorbent assay (ELISA). Levels of IL-2 and IFN- $\gamma$ were determined using the ELISA kit procured from Diaclone SAS, (Besancon, France), according to the manufacturer's protocol.

Statistical analysis. All results were expressed as the mean \pm standard deviation of the group. Statistical comparisons were performed using one-way analysis of variance and Tukey's post-hoc test. PRISM software (version 7; GraphPad Software Inc., La Jolla, CA, USA) was used for the analyses. $\mathrm{P}<0.05$ was considered to indicate a statistically significant difference.

\section{Results}

Changes in body and liver weights. The body weight of the mice was measured at weeks 0 and 12 of the experimental protocol. The mean weight of the groups was adjusted, without any significant difference present between groups at week 0 . At the end of the experimental period of 12 weeks, all the groups were weighed and a significant difference $(\mathrm{P}=0.031)$ of $23.3 \%$ decrease in the body weight was identified in the DN group compared with the control group mice (Table I). Similarly, a significant increase in the liver weight $(37.5 \%$; $\mathrm{P}=0.021)$ was noted in the DN group compared with the control. Although the decrease in body weight and increase in liver weight of mice recovered towards normal levels during LUT treatment, no significant difference was noted.

Activity of marker enzymes in the plasma and liver. Marker enzymes, such as aspartate aminotransferase (AST) and alanine aminotransferase (ALT) were assayed in the plasma and liver tissues of the experimental mice (Figs. 1 and 2). Significant differences were noted between groups $(\mathrm{P}=0.042)$, and the levels of AST and ALT in the plasma were 109.8 and $148.3 \%$, respectively, and the levels of AST and ALT in the liver tissue were 55.5 and $61.3 \%$, respectively. All these alterations returned to the normal levels during LUT treatment.

Activity of enzymatic antioxidants in the liver. Enzymatic antioxidants, including SOD and CAT, defend against oxidative stress (20). The activity of the enzymatic antioxidants SOD and CAT was significantly decreased by 38.6 and $12.5 \%$, respectively, in DN intoxication (Table II). During LUT treatment, the decrease in activity was restored to normal. No 
Table I. Body and liver weights of mice in different experimental groups.

\begin{tabular}{lrrrr}
\hline Specification & Control & LUT & DN & DN+LUT \\
\hline Body weight, g & & & & \\
Week 0 & $18.9 \pm 1.10^{\mathrm{a}}$ & $19.2 \pm 0.80^{\mathrm{a}}$ & $19.1 \pm 1.40^{\mathrm{a}}$ & $19.4 \pm 0.60^{\mathrm{a}}$ \\
Week 12 & $24.5 \pm 1.90^{\mathrm{a}}$ & $25.9 \pm 3.70^{\mathrm{a}}$ & $18.8 \pm 1.90$ & $21.5 \pm 2.40^{\mathrm{a}}$ \\
Liver weight, g & $0.8 \pm 0.01^{\mathrm{a}}$ & $0.8 \pm 0.02^{\mathrm{a}}$ & $1.1 \pm 0.06$ & $1.0 \pm 0.09^{\mathrm{a}}$ \\
\hline
\end{tabular}

Results are expressed as the mean \pm standard deviation of 6 mice. Means within the same row with different superscripts differ significantly $\left({ }^{\mathrm{a}} \mathrm{P} 0\right.$.05). LUT, luteolin; DN, diethylnitrosamine.

Table II. Activity of SOD and CAT in the liver of mice in different experimental groups.

\begin{tabular}{lllll}
\hline Specification & Control & LUT & DN & DN+LUT \\
\hline SOD, U/mg protein & $45.9 \pm 3.8^{\mathrm{a}}$ & $44.8 \pm 4.6^{\mathrm{a}}$ & $28.2 \pm 4.9$ & $35.3 \pm 5.2^{\mathrm{a}}$ \\
CAT, U/mg protein & $31.9 \pm 2.5^{\mathrm{a}}$ & $32.4 \pm 3.8^{\mathrm{a}}$ & $27.9 \pm 3.1$ & $31.3 \pm 1.8^{\mathrm{a}}$ \\
\hline
\end{tabular}

Results are expressed as the mean \pm standard deviation of 6 mice. Means within the same row with different superscripts differ significantly $\left({ }^{a} \mathrm{P} \leq 0.05\right)$. LUT, luteolin; DN, diethylnitrosamine; SOD, superoxide dismutase; CAT, catalase.

Table III. Levels of plasma LPO and GSH in mice in different experimental groups.

\begin{tabular}{lcrrr}
\hline Specification & Control & LUT & DN & DN+LUT \\
\hline LPO, mM TBARS/ml & $11.48 \pm 1.10^{\mathrm{a}}$ & $12.73 \pm 1.10^{\mathrm{a}}$ & $92.82 \pm 6.90$ & $61.32 \pm 7.90^{\mathrm{a}}$ \\
GSH, mg/dl & $1.95 \pm 0.09^{\mathrm{a}}$ & $2.08 \pm 0.06^{\mathrm{a}}$ & $0.67 \pm 0.05$ & $1.17 \pm 0.15^{\mathrm{a}}$
\end{tabular}

Results are expressed as the mean \pm standard deviation of 6 mice. Means within the same row with different superscripts differ significantly ( ${ }^{\mathrm{a}} \mathrm{P} \leq 0.05$ ). LUT, luteolin; DN, diethylnitrosamine; LPO, lipid peroxidation; GSH, reduced glutathione; TBARS, thiobarbituric acid reactive substances.

Table IV. Levels of liver LPO and GSH in mice in different experimental groups.

\begin{tabular}{|c|c|c|c|c|}
\hline Specification & Control & LUT & $\mathrm{DN}$ & $\mathrm{DN}+\mathrm{LUT}$ \\
\hline LPO, nM TBARS/g tissue & $30.8 \pm 1.60^{\mathrm{a}}$ & $31.7 \pm 2.10^{a}$ & $89.3 \pm 5.20$ & $71.5 \pm 4.60$ \\
\hline $\mathrm{GSH}, \mathrm{mg} / \mathrm{g}$ tissue & $4.6 \pm 0.79^{\mathrm{a}}$ & $4.9 \pm 0.57^{\mathrm{a}}$ & $2.6 \pm 0.09$ & $3.8 \pm 0.19^{\mathrm{a}}$ \\
\hline
\end{tabular}

Results are expressed as the mean \pm standard deviation of 6 mice. Means within the same row with different superscripts differ significantly $\left({ }^{a} \mathrm{P} \leq 0.05\right)$. LUT, luteolin; DN, diethylnitrosamine; LPO, lipid peroxidation; GSH, reduced glutathione; TBARS, thiobarbituric acid reactive substances.

significant difference was identified between the activity in the group administered with LUT alone and the group treated with DN and LUT.

Level of LPO for oxidative stress. LPO was assessed by the levels of the thiobarbituric acid reactive substance malondialdehyde in the plasma and tissue, which was used to quantitate the oxidative stress due to $\mathrm{DN}$ administration (Tables III and IV). Significant increases $(\mathrm{P}=0.016)$ in the level of LPO of $\sim 7$-fold and $\sim 2$-fold were identified in the plasma and liver tissues, respectively. These increases were returned to the normal level during LUT treatment.
Levels of GSH indicated the redox status. GSH level indicates the redox status and health of cells. In the present study, 65.6 and $43.5 \%$ decreases in the GSH level were identified in the plasma and liver, respectively, during DN intoxication, which was then significantly $(\mathrm{P}=0.036)$ normalized during LUT treatment (Tables III and IV). Notably, a significant increase $(6.7 \%$; $\mathrm{P}=0.027)$ in the plasma GSH level was noted in the group administered with LUT alone.

AFP levels in the plasma. AFP is a circulating tumor marker. A 12-fold increase in the AFP level in the plasma of mice treated with DN indicated the progression of neoplastic disease in the 


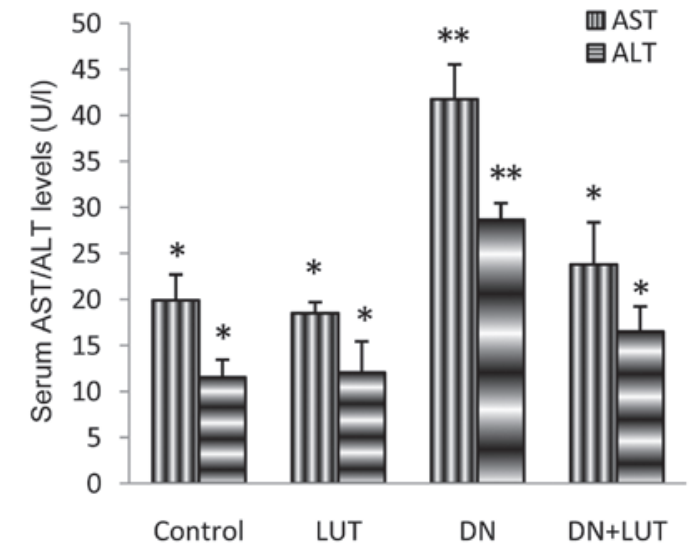

Figure 1. Effect of LUT on plasma marker enzymes in DN-intoxicated mice. Results are expressed as the mean \pm standard deviation for 6 mice. Mean values in different groups with different numbers of arterisks (same type) differ significantly $(\mathrm{P}<0.05)$. LUT, luteolin; DN, diethylnitrosamine; AST, aspartate aminotransferase; ALT, alanine aminotransferase. ${ }^{*} \mathrm{P}<0.05$ and ${ }^{* * *} \mathrm{P}<0.01$.

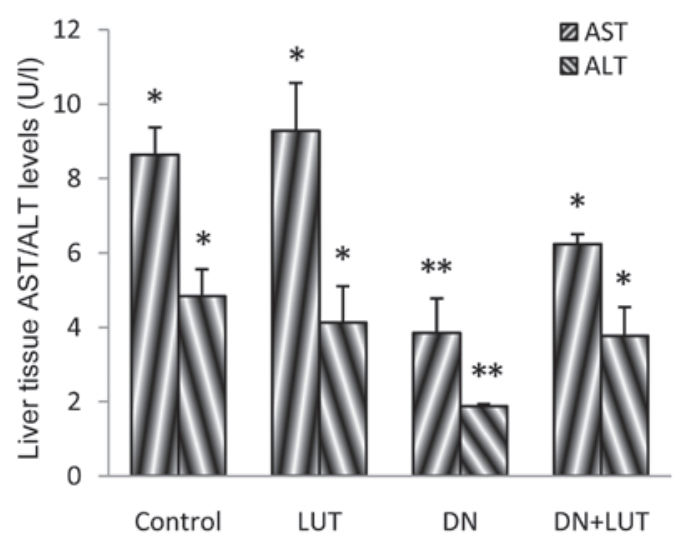

Figure 2. Effect of LUT on liver marker enzymes in DN-intoxicated mice. Results are expressed as the mean \pm standard deviation for 6 mice. Mean values in different groups with different numbers of arterisks (same type) differ significantly $(\mathrm{P}<0.05)$. LUT, luteolin; DN, diethylnitrosamine; AST, aspartate aminotransferase; ALT, alanine aminotransferase. ${ }^{*} \mathrm{P}<0.05$ and ${ }^{* *} \mathrm{P}<0.01$.

animals (Fig. 3). The significant decrease of the AFP level during LUT treatment indicated the suppression of the disease.

Levels of inflammatory markers in the plasma. IL-2 and IFN $-\gamma$ are inflammatory markers that indicate the progression of disease and the status of the immune response of the host. A significant $(\mathrm{P}=0.037) 31.2 \%$ decrease in the IL-2 level and $36.2 \%$ increase in the IFN- $\gamma$ level were identified during DN intoxication when compared with the control (Fig. 4). The return of these alterations to the normal level during LUT treatment indicated the protective effect of LUT against tumor development.

\section{Discussion}

LUT has numerous biological roles in prophylactic and therapeutic modes of treatment. Alterations in the body and liver weights during $\mathrm{DN}$ intoxication indicate the progression of disease, and the reversal of DN intoxication resulted in the normalization of the body and liver weights. Pathological

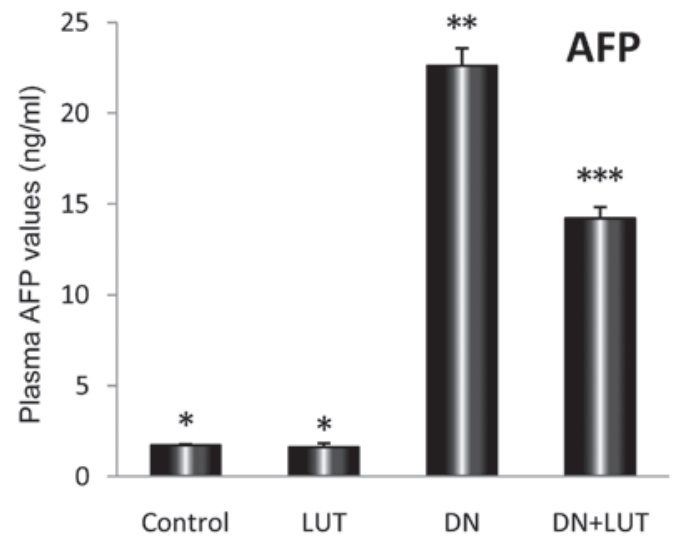

Figure 3. Effect of LUT on plasma AFP in DN-intoxicated mice. Results are expressed as the mean \pm standard deviation for 6 mice. Mean values in different groups with different numbers of arterisks (same type) differ significantly $(\mathrm{P}<0.05)$. LUT, luteolin; AFP, $\alpha$-fetoprotein; DN, diethylnitrosamine. ${ }^{*} \mathrm{P}<0.05,{ }^{* *} \mathrm{P}<0.01$ and ${ }^{* * * *} \mathrm{P}<0.001$.

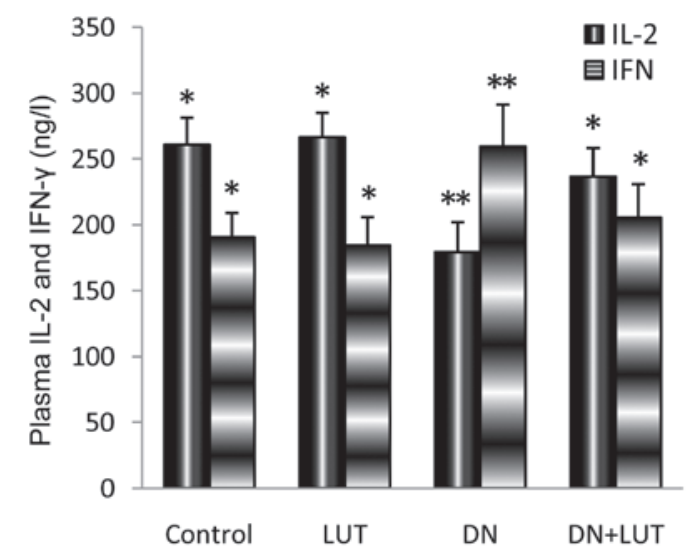

Figure 4. Effect of LUT on plasma IL-2 and IFN- $\gamma$ in DN intoxicated mice. Results are expressed as the mean \pm standard deviation for 6 mice. Mean values in different groups with different numbers of arterisks (same type) differ significantly $(\mathrm{P}<0.05)$. LUT, luteolin; DN, diethylnitrosamine; IFN- $\gamma$, interferon- $\gamma$; IL-2, interleukin-2. ${ }^{*} \mathrm{P}<0.05$ and ${ }^{* *} \mathrm{P}<0.01$.

changes leading to increased production or decreased scavenging of free radicals may play a crucial role in tissue injury (21). The cellular mechanism of toxicity produced by DN may involve the marker enzymes (AST and ALT), and may result in the elevation of these enzymes in the plasma and decrease of the marker enzymes in the liver $(8,22)$. A significant normalization of the level of the marker enzymes during LUT treatment may be beneficial, as indicated by the protective role of the drug. Increased free radical production stimulates LPO and is the source of the degradation of DNA, lipids and carbohydrates (23). Numerous flavonoid aglycones have been shown to have chain-breaking antioxidant properties and to quench free radicals, thereby exerting protective effects on macromolecules (24). The cellular level of total GSH content determines the total redox potential of a cell (25). The restoration of a protective level of GSH and suppression of LPO in the plasma and liver tissues depicts the protective role of LUT against ROS and oxidative damage. This is consistent with previous studies $(12,16,20)$. Notably, a significant increase in the plasma GSH level was identified in the group that was 
administered with LUT alone, which indicates the increased health of the normal animals, due to the antioxidant efficacy of GSH.

Enzymatic antioxidants, such as SOD and CAT, and the non-protein thiol GSH are involved in primary cellular antioxidant defense mechanisms (20). Antioxidant enzymes, including SOD and CAT, may either directly detoxify the ROS produced or facilitate antioxidant reactions using GSH as a reducing agent (26). CAT is responsible for the decomposition of peroxides in a cell (27). In the present study, disturbances in the balance between the ROS levels and expression of antioxidant defenses were indicated and LUT was revealed to efficiently resolve this imbalance.

AFP is an oncoglycoprotein of unknown function that is more prevalent in Italian patients with cirrhosis and hepatocellular carcinoma. The level of AFP is considered to differentiate HCC from chronic liver disease (28). Additionally, AFP mRNA acts as a circulating marker that may be used as a more reliable indicator of liver cancer $(29,30)$. The high level of AFP present during DN administration was significantly decreased during the subsequent LUT treatment, and this reduction demonstrated the protective effects of LUT.

IL-2 is an important inflammatory cytokine. An increased level of IL-2 is involved in efficient cellular immune function (31). Previous studies have shown that a locally-secreted high level of IL-2 generates fewer side effects compared with a systemically administered high dose of IL-2 $(31,32)$. In the present study, the low level of IL-2 present during the formation of liver lesions was significantly increased during treatment with LUT. Due to this increase in the IL-2 level, treatment with LUT may be beneficial for the development of the immune response against the tumor in vivo. IFN- $\gamma$ is one of the important inflammatory cytokines that trigger liver damage during various stresses, and the depletion of IFN- $\gamma$ may result in a marked reduction in injury and inflammation (33). In the present study, treatment with LUT decreased the elevated level of IFN- $\gamma$ and thereby suppressed the inflammatory response, which is consistent with previously studies in the literature (34). Significant reversal of the pathological alterations during LUT treatment indicates the treatment efficiency of LUT and may aid the triggering of the host immune response against the tumor. However, additional information on the underlying mechanism of the suppression of inflammatory cytokines and protective action of LUT is required from additional investigation.

\section{References}

1. Altekruse SF, McGlynn KA and Reichman ME: Hepatocellular carcinoma incidence, mortality and survival trends in the United States from 1995 to 2005. J Clin Oncol 27: 1485-1491, 2009.

2. Fattovich G, Stroffolini T, Zagni I and Donato F: Hepatocellular carcinoma and cirrhosis: Incidence and risk factors. Gastroenterology 127 (5 Suppl 1): S35-S50, 2004.

3. Faloppi L, Scartozzi M, Maccaroni E, Di Pietro Paolo M, Berardi R, Del Prete M and Cascinu S: Evolving strategies for the treatment of hepatocellular carcinoma: From clinical-guided to molecularly-taylored therapeutic options. Cancer Treat Rev 37: 169-177, 2011.

4. Borzio M, Fargion S, Borzio F, Fracanzani AL, Croce AM, Stroffolini T, Oldani S, Cotichini R and Roncalli M: Impact of large regenerative, low grade and high grade dysplastic nodules in hepatocellular carcinoma development. J Hepatol 39: 208-214, 2003 .
5. Pawlik TM, Poon RT, Abdalla EK, Ikai I, Nagorney DM, Belghiti J, Kianmanesh R, Ng IO, Curley SA, Yamaoka Y, et al: Hepatectomy for hepatocellular carcinoma with major portal or hepatic vein invasion: Results of a multicenter study. Surgery 137: 403-410, 2005.

6. Irtan S, Chopin-Laly X, Ronot M, Faivre S, Paradis V and Belghiti J: Complete regression of locally advanced hepatocellular carcinoma induced by sorafenib allowing curative resection. Liver Int 31: 740-743, 2011.

7. Petersen FB, Buckner CD, Appelbaum FR, Clift RA, Sanders JE, Bensinger WI, Storb R, Witherspoon RP, Sullivan KM and Bearman SI: Busulfan, cyclophosphamide and fractionated total body irradiation as a preparatory regimen for marrow transplantation in patients with advanced hematological malignancies: A phase I study. Bone Marrow Transplant 4: 617-623, 1989.

8. Senthilkumar S, Devaki T, Manohar BM and Babu MS: Effect of squalene on cyclophosphamide-induced toxicity. Clin Chim Acta 364: 335-342, 2006

9. Clemens MR, Ladner C, Schmidt H, Ehninger G, Einsele H, Bühler E, Waller HD and Gey KF: Decreased essential antioxidants and increased lipid hydroperoxides following high-dose radiochemotherapy. Free Radic Res Commun 7: 227-232, 1989.

10. Hosakote YM, Liu T, Castro SM, Garofalo RP and Casola A: Respiratory syncytial virus induces oxidative stress by modulating antioxidant enzymes. Am J Respir Cell Mol Biol 41: 348-357, 2009.

11. Zhang Y, Li J, Cao L, Xu W and Yin Z: Circulating tumor cells in hepatocellular carcinoma: Detection techniques, clinical implications and future perspectives. Semin Oncol 39: 449-460, 2012.

12. Lopez-Lazaro M: Distribution and biological activities of the flavonoid luteolin. Mini Rev Med Chem 9: 31-59, 2009.

13. Cai X, Lu W, Ye T, Lu M, Wang J, Huo J, Qian S, Wang X and Cao P: The molecular mechanism of luteolin-induced apoptosis is potentially related to inhibition of angiogenesis in human pancreatic carcinoma cells. Oncol Rep 28: 1353-1361, 2012.

14. Merfort I, Heilmann J, Hagedorn-Leweke U and Lippold BC: In vivo skin penetration studies on chamomile flavones. Pharmazie 49: 509-511, 1994

15. Plaschke K: Composition comprising one or more flavonoids, method of obtaining such composition and use there of as UV-absorbing agent. US Patent 6,409,996 B1. Filed November 19, 1998; issued June 25, 2002.

16. Graefe EU, Derendorf H and Veit M: Pharmacokinetics and bioavailability of the flavonols quercetin in humans. Int J Clin Pharmacol Ther 37: 219-233, 1999.

17. Ohkawa H, Ohishi $\mathrm{N}$ and Yagi K: Assay for lipid peroxides in animal tissues by thiobarbituric acid reaction. Anal Biochem 95: 351-358, 1979.

18. Ellman GL: Tissue sulfhydryl groups. Arch Biochem Biophys 82: 70-77, 1959.

19. Morota K, Komori M, Fujinami R, Yamada K, Kuribayashi K, Watanabe N, Sokoll LJ, Elliott D, Chan DW, Martens F, et al: Improvement and multicenter evaluation of the analytical performance of an automated chemiluminescent immunoassay for alpha fetoprotein. Int J Biol Markers 27: 39-46, 2012.

20. Rice-Evans CA, Miller NJ and Paganga G: Structure-antioxidant activity relationships of flavonoids and phenolic acids. Free Radic Biol Med 20: 933-956, 1996.

21. Halliwell B and Gutteridge JM: Lipid peroxidation, oxygen radicals, cell damage and antioxidant therapy. Lancet 1: 1396-1397, 1984

22. Senthilkumar S, Ebenezar KK, Sathish V, Yogeeta S and Devaki T: Modulation of tissue defense system by squalene in cyclophosphamide induced toxicity in rats. Arch Med Sci 2: 94-100, 2006

23. Quinlan GJ and Gutteridege JM: Hydroxyl radical generation by the tetracycline antibodies with free radical damage to DNA, lipids, carbohydrates in the presence of iron and copper salts. Free Radic Biol Med 5: 341-348, 1988.

24. Van Poppel G, Verhoeven DT, Verhagen H and Goldbohm RA: Brassica vegetables and cancer prevention. Epidemiology and mechanisms. Adv Exp Med Biol 472: 159-168, 1999.

25. Østergaard H, Tachibana $\mathrm{C}$ and Winther JR: Monitoring disulfide bond formation in the eukaryotic cytosol. J Cell Biol 166: 337-345, 2004.

26. Senthilkumar S, Yogeeta SK, Subashini R and Devaki T: Attenuation of cyclophosphamide induced toxicity by squalene in experimental rats. Chem Biol Interact 160: 252-260, 2006.

27. Tafazoli S and O'Brien PJ: Peroxidases: A role in the metabolism and side effects of drugs. Drug Discov Today 10: 617-625, 2005. 
28. Trevisani F, D'Intino PE, Morselli-Labate AM, Mazzella G, Accogli E, Caraceni P, Domenicalli M, De Notariis S, Roda E and Bernardi M: Serum alpha-fetoprotein for diagnosis of hepatocellular carcinoma in patients with chronic liver disease: Influence of HBsAg and anti- HCV status. J Hepatol 34: 570-575, 2001.

29. Wright LM, Kreikemeier JT and Fimmel CJ: A concise review of serum markers for hepatocellular cancer. Cancer Detect Prev 31: 35-44, 2007.

30. Chiappini F: Circulating tumor cells measurements in hepatocellular carcinoma. Int J Hepatol 2012: 684802, 2012.
31. Yang Gui-zhen: Boundary immunology. Beijing, Science Press, 2002.

32. Tagawa M: Cytokine therapy for cancer. Curr Pharm Des 6: 681-699, 2000.

33. Kusters S, Gantner F, Künstle G and Tiegs G: Interferon-ã plays a critical role in $\mathrm{T}$ cell-dependent liver injury in mice initiated by concanavalin A. Gastroenterology 111: 462-471, 1996.

34. Haqqi TM, Anthony DD, Gupta S, Ahmad N, Lee MS, Kumar GK and Mukhtar H: Prevention of collagen-induced arthritis in mice by a polyphenolic fraction from green tea. Proc Natl Acad Sci USA 96: 4524-4529, 1999. 